\begin{tabular}{|c|c|}
\hline Title & Effects of Cathode Catalyst Layer Structure and Properties Dominating Polymer Electrolyte Fuel Cell Performance \\
\hline Author(s) & Tabe, Y utaka; Nishino, Masay oshi; Takamatsu, Hiroyuki; Chikahisa, Takemi \\
\hline Citation & $\begin{array}{l}\text { Journal of the Electrochemical Society, 158(10), B1246-B1254 } \\
\text { https://doi.org/10.1149/.3624606 }\end{array}$ \\
\hline Issue Date & $2011-08-12$ \\
\hline Doc URL & http:/hdl .handle.net/2115/47104 \\
\hline Rights & $\begin{array}{l}\text { () The Electrochemical Society, Inc. 2011. All rights reserved. Except as provided under U.S. copyright law, this work } \\
\text { may not be reproduced, resold, distributed, or modified without the express permission of The Electrochemical Society } \\
\text { (ECS). The archival version of this work was published in J. Electrochem. Soc., 158(10), pp. B1246-B1254 (2011) }\end{array}$ \\
\hline Type & article \\
\hline File Information & JES158-10_B1246-B1254.pdf \\
\hline
\end{tabular}

Instructions for use 


\title{
Effects of Cathode Catalyst Layer Structure and Properties Dominating Polymer Electrolyte Fuel Cell Performance
}

\author{
Yutaka Tabe, ${ }^{*, z}$ Masayoshi Nishino, Hiroyuki Takamatsu, and Takemi Chikahisa* \\ Division of Energy and Environmental Systems, Graduate School of Engineering, Hokkaido University, \\ Sapporo 060-8628, Japan
}

\begin{abstract}
Transport of electrons, protons, and oxygen are necessary for the cathode reactions in polymer electrolyte membrane fuel cells, and achieving the optimum structure of the electrode catalyst layer and the efficient transport of reactants is an effective avenue to reduce the use of platinum catalyst. This study applied three-phase boundary and cathode catalyst layer models to understand details of optimally efficient structures for the transport of reaction components. The factors dominating the effects of the catalyst layer structure and the properties identified in this manner are investigated using the models. Additionally, equations of evaluation are developed to evaluate the effects of the structure and the properties on the cell performance, and the effectiveness of the developed equations is confirmed by a comparison of the results calculated by the equations with the model simulations. From these results, the structure of the porosity, the catalyst layer, and the polymer electrolyte thicknesses, that are optimum for the gas transport and proton conduction, are determined. It is found that the solubility of oxygen in the polymer is one of the dominant factors in the processes of the cathode catalyst layer, and that increasing the solubility is highly effective to reduce the need for platinum. (C) 2011 The Electrochemical Society. [DOI: 10.1149/1.3624606] All rights reserved.
\end{abstract}

Manuscript submitted April 6, 2011; revised manuscript received July 20, 2011. Published August 12, 2011. This was Paper 617 presented at the Las Vegas, Nevada Meeting of the Society, October 10-15, 2010. Publisher error corrected August $23,2011$.

The polymer electrolyte membrane fuel cell (PEFC) has attracted increasing attention as a power source with high efficiency and clean emissions for automobiles and stationary distributed power supply systems. Many issues still remain for the practical use of PFEC and one such is the high cost due to expensive components such as the platinum catalyst and membrane required for efficient operation. In particular, the catalyst layer of the cathode requires much platinum because of the slowness of the oxygen reduction reaction in PEFCs. Therefore, the cost reductions that would be possible by reduced platinum usage must be balanced by the need to maintain high performance. Because electrons, protons, and oxygen are necessary for the cathode reaction, ensuring the optimum structure of the electrode catalyst layer and the efficient transport of reactants would be effective to reduce the usage of platinum catalyst.

Determination of optimum compositions and structures of the catalyst layer of PEFCs has been conducted using time-consuming and expensive experiments, as well as computational modeling approaches to elucidate details of the processes in the catalyst layer have been reported. ${ }^{1-14}$ Catalyst layer models are classified into three categories: pseudo-homogeneous film models, ${ }^{1-3}$ agglomerate models, ${ }^{4-11}$ and pore-scale models. ${ }^{12-14}$ The pseudo-homogeneous film models assume that the catalyst layer is a porous matrix comprised of Nafion, platinum and carbon in a locally homogeneous configuration. This approach has been used to discuss the optimum Nafion content and platinum loading ${ }^{1,2}$ and a model that links the spatial distribution of the various processes with water handling capabilities and current voltage performance was presented, ${ }^{3}$ however these models are inadequate to evaluate the transport phenomena around the three-phase boundary with the polymer electrolyte (the Nafion in these references) for the proton transfer, the platinum for the catalysis, and the carbon for the electron transfer. The agglomerate models assume that the platinum-supported carbons form agglomerates with the polymer electrolyte, and the carbon agglomerate is coated fully or partially with an additional polymer electrolyte. This model can allow for limitations arising from proton migration in the active layer and oxygen diffusion in the agglomerates, ${ }^{4}$ and the effects of the catalyst layer structure including Nafion and platinum. ${ }^{5-10}$ The pore-scale models have employed direct numerical simulation of the oxygen, water, and proton transport through a catalyst layer microstructure, while the agglomerate models use an approximate macroscopic approach. The pore-scale approach is more useful to simulate cell performance in actual catalyst layer structures, ${ }^{12-14}$ but it is limited by the complicated and

\footnotetext{
* Electrochemical Society Active Member.

${ }^{\mathrm{z}}$ E-mail: tabe@eng.hokudai.ac.jp
}

difficult reconstruction of the catalyst layer required and the huge amount of computation that this kind of simulation involves. These models all have both strengths and weaknesses, and wide ranging research with the various models is necessary to elucidate further details of the optimum structure to achieve optimum platinum utilization.

The objective of this study is to establish the dominant effects of the structure and the properties in the cathode catalyst layer on the performance of PEFCs and to elucidate the catalyst layer structures that are advantageous for an efficient utilization of cathode platinum. Here, we apply a three-phase boundary model and a cathode catalyst layer model based on a simplified agglomerate concept. Using the models, the effects of the catalyst layer structure and the properties on the cell performance are investigated. Additionally, equations to estimate these effects and to understand the factor dependence simply are developed, and the applicability of the equations evaluating the effects is confirmed by a comparison of the results calculated based on the equations and model simulations. From the analyses by the model simulation and the evaluating equations, the factors playing dominant roles in the cell performance are isolated, and effective strategies for reducing the platinum usage in the structures and the related properties of the cathode catalyst layer are discussed.

\section{Model Description}

Three-phase boundary and catalyst layer models.-This study analyzes only the phenomena in the cathode catalyst layer as the overpotential in the cathode is much larger than that in the anode. The study attempts to identify the factors dominating the effects of the structure and the properties in the cathode catalyst layer on the cell performance. To achieve this, the simplified agglomerate model in Fig. 1a was applied as the model for the cathode three-phase boundary, which is the reaction site of the platinum surface where electrons, protons, and reaction gases can be supplied and removed. It was assumed that a platinum-supported carbon agglomerate is covered with a uniform thick polymer electrolyte and that the cathode reaction occurs only due to dissolved oxygen in the polymer at the carbon agglomerate surface. Further that all of the platinum (Pt) catalyst contributes to the reaction and that the mass transport losses inside the carbon agglomerate are negligible. This leads to a slight overestimation of the cell performance and it is necessary to establish a better structural model for the carbon agglomerate and to set the properties of the agglomerate suitably for precise performance predictions. Further, the oxygen dissolution rate from the gas phase into the polymer, which has recently been noted as an important 


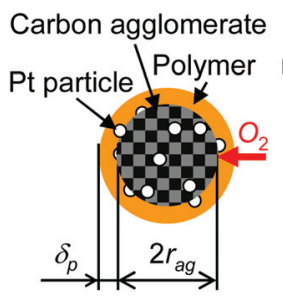

(a) Three-phase boundary mode
Polymer membrane

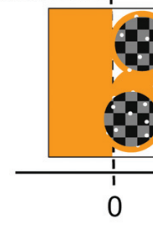

(b) Cathode catalyst layer model
Figure 1. (Color online) Schematic outline of the models.

resistance factor in the mass transport of the cathode catalyst layer, ${ }^{15}$ must be considered for the quantitative predictions of the cell performance. Figure $1 \mathrm{~b}$ shows a schematic diagram of the cathode catalyst layer model, here identical carbon agglomerates with polymer electrolyte film (as in Fig. 1a) are dispersed uniformly in the catalyst layer. In the $z$-axis, the thicknesses, direction, the origin and $z=\delta_{C L}$ are the interfaces between the polymer electrolyte membrane (PEM) and the catalyst layer, and between the catalyst layer and the gas diffusion layer (GDL). In this one-dimensional model of the cathode catalyst layer, the surface areas of the agglomerates and platinum catalysts were calculated using the number of the agglomerates per unit volume in the catalyst layer, $n_{a g}$. The phenomena in the cathode catalyst layer were analyzed under the following assumptions:

(a) the catalyst layer and the GDL are isothermal, isobaric, and at a steady state condition,

(b) the electric potential of the carbon agglomerate is constant,

(c) the generated water is in the gas phase.

These assumptions have been conventionally applied in catalyst layer simulations, and it was confirmed that simulations using these assumptions are able to predict experimental results. ${ }^{15}$ Further, a model of the three principal wetting states in the cathode catalyst layer has been proposed, here liquid water appears in the secondary pores above $2.5 \mathrm{~A} \mathrm{~cm}^{-2}$. 3 To accommodate this, it is necessary to predict the temperature distribution and the formation behavior of the liquid water film in the catalyst layer in the considerations of the liquid water effects on the cell performance, and this is an important issue for the next step. This study assumes a uniform thickness of the polymer electrolyte around the agglomerate and the effect of non-uniform polymer films is also an issue to be considered in further refinements of the simulations.

Formulation of models.-The oxygen reduction reaction $\left(\mathrm{O}_{2}+4 \mathrm{H}^{+}+4 e^{-} \rightarrow 2 \mathrm{H}_{2} \mathrm{O}\right)$ occurs at the carbon agglomerate surface, where the dissolved oxygen and the protons in the polymer electrolyte, and the electrons in the carbon are consumed. Oxygen was assumed to diffuse in the polymer according to Fick's law of diffusion, and spherical diffusion of the dissolved oxygen in the polymer is expressed by the following equation

$$
J_{O_{2}}^{p}(r)=-C_{p} D_{O_{2}}^{p} \frac{\partial x_{O_{2}}^{p}}{\partial r}
$$

Here, $C_{p}$ is the total molar concentration of the polymer, and $D_{\mathrm{O}_{2}}^{p}$ and $x_{\mathrm{O}_{2}}^{p}$ are the diffusion coefficient and the mole fraction of the oxygen in the polymer. No oxygen is consumed in the polymer, and the total molar concentration of the polymer and the diffusion coefficient are approximately constant. The oxygen diffusion flux in the polymer, $J_{\mathrm{O}_{2}}^{p}$, is obtained by the following equation

$$
J_{O_{2}}^{p}(r)=-D_{O_{2}}^{p} \frac{\left(C_{O_{2}}^{S}-C_{O_{2}}^{*}\right) r_{a g}\left(r_{a g}+\delta_{p}\right)}{\delta_{p} r^{2}}
$$

with $C_{O_{2}}^{S}$ and $C_{O_{2}}^{*}$ the oxygen concentrations in the polymer at the gas phase $\left(r=r_{a g}+\delta_{p}\right)$ and the carbon agglomerate $\left(r=r_{a g}\right)$ interfaces. The oxygen diffusion flux in the polymer equals the rate of consumption by the cathode reduction reaction at the reaction area $\left(r=r_{a g}\right)$, and the oxygen concentration was assumed to be in an equilibrium state obeying Henry's law, as in Eqs. 3 and 4

$$
\begin{gathered}
J_{O_{2}}^{p}\left(r_{a g}\right)=-k_{O R R} C_{O_{2}}^{*} \\
\gamma=C_{O_{2}}^{S} / C_{O_{2}}^{g}
\end{gathered}
$$

Here, $k_{O R R}$ is the reaction constant of the oxygen reduction reaction per the unit effective reaction area, $C_{O_{2}}^{g}$ is the oxygen concentration in the gas phase at the interface of the polymer, and $\gamma$ is the ratio of the oxygen concentration in the polymer to that in the gas phase. From Eqs. 2-4, the oxygen diffusion flux at the carbon agglomerate surface, $J_{O_{2}}^{a g}$, can be expressed by the oxygen concentration in the gas phase at the polymer surface, $\mathrm{C}_{\mathrm{O}_{2}}^{g}$, as follows.

$$
J_{O_{2}}^{a g}=-C_{O_{2}}^{g} \gamma\left[\frac{r_{a g} \delta_{p}}{\left(r_{a g}+\delta_{p}\right) D_{O_{2}}^{p}}+\frac{1}{k_{O R R}}\right]^{-1}
$$

The first and second terms in the bracket on the right hand side represent the oxygen diffusion resistance in the polymer and the reaction resistance at the reaction site of the agglomerate surface, respectively. The reaction constant of the oxygen reduction reaction, $k_{\text {ORR }}$, was given by a simplified Butler-Volmer expression, ${ }^{11}$ Eq. 6

$$
k_{O R R}=\frac{A i_{0}}{4 F C_{0, O_{2}}} \exp \left(-\frac{2.303 \eta}{b}\right)
$$

Here, $A$ is the area ratio of the reaction site of the platinum surface to the surface of the carbon agglomerate $\left(A=r_{P t}^{2} n_{P t} / r_{a g}^{2} n_{a g}\right), i_{0}$ is the exchange current density at the standard oxygen concentration, $F$ is Faraday's constant, $C_{0, O_{2}}$ is the standard oxygen concentration in the polymer, $\eta$ is the cathode overpotential, and $b$ is the Tafel slope. And, $n_{P t}\left(=3 m_{P t} / 4 \pi \rho_{P t} r_{P t}^{3} \delta_{C L}\right)$ and $n_{a g}\left(=3\left(1-\varepsilon_{C L}\right) / 4 \pi\right.$ $\left.\left(r_{a g}+\delta_{p}\right)^{3}\right)$ are the number densities of platinum particles and carbon agglomerates, and these can be calculated from the supported platinum mass per unit area, $m_{P t}$, the platinum density, $\rho_{P t}$, the radius of catalyst particles, $r_{P t}$, the porosity of the catalyst layer, $\varepsilon_{C L}$, and other parameters.

The proton current in the catalyst layer is generated by the oxygen consumption obtained from Eq. 5, and the change in the proton current density, $i_{H+}$, is satisfied with the following balance

$$
\frac{d i_{H^{+}}(z)}{d z}=4 F \cdot n_{a g} S_{a g} J_{O_{2}}^{a g}
$$

Here, $S_{a g}$ is the surface area of one carbon agglomerate $\left(=4 \pi r_{a g}^{2}\right)$. The change in the cathode overpotential, $\eta$, corresponds to the voltage decrease due to the proton transport, and it is expressed by the following equation, using the proton current density, $i_{H+}$, and the effective proton conductivity, $\kappa^{e f f, C L}$.

$$
\frac{d \eta(z)}{d z}=\frac{i_{H^{+}}}{\kappa^{e f f, C L}}
$$

As will be shown in the Section of results and discussion, the distributions of the cathode reaction rate and the cathode overpotential can be calculated by setting $\eta\left(\delta_{C L}\right)$ as a boundary condition at the interface between the catalyst layer and the GDL, together with $i_{H+}\left(\delta_{C L}\right)=0$. Here, the overall reaction rate in the catalyst layer corresponds to the proton current density at the interface between the catalyst layer and the PEM, $i_{H+}(0)$, and the overall rates of oxygen consumption and water generation in the entire catalyst layer are $i_{H+}(0) / 4 F$ and $i_{H+}(0) / 2 F$. 
The oxygen gas transfers into the catalyst layer through the pores and dissolves in the polymer electrolyte surrounding the agglomerates, whereas the generated water on the agglomerate surfaces transfers through the polymer and is discharged in the gas phase in the pore. Because the number of moles of generated water is twice that of the consumed oxygen in the oxygen reduction reaction, the reaction induces advection from the PEM to the GDL in the pores of the cathode catalyst layer. Assuming that the total mole concentration in the pores, $C^{g}$, is constant, the continuity equation is expressed by Eq. 9 and the conservation equations for the oxygen and the water gas by Eq. 10 in the pores of the catalyst layer using the advection velocity, $v$

$$
\begin{gathered}
\varepsilon_{C L} C^{g} \frac{d v(z)}{d z}=\dot{\omega}_{O_{2}}+\dot{\omega}_{H_{2} O} \\
\varepsilon_{C L} \frac{d\left(C_{i}^{g}(z) v(z)\right)}{d z}=D_{i}^{e f f, C L} \frac{d^{2} C_{i}^{g}(z)}{d z^{2}}+\dot{\omega}_{i}
\end{gathered}
$$

Where $-\dot{\omega}_{\mathrm{O}_{2}}$ and $\dot{\omega}_{\mathrm{H}_{2} \mathrm{O}}$ are the rates of oxygen consumption and water generation; $C_{i}^{g}$ and $D_{i}^{\text {eff, }, C L}$ are the concentration and the effective diffusion coefficient of species $i$ in the gas phase of the catalyst layer. In this one-dimensional model of the cathode catalyst layer, the distributions of the gas concentrations between the polymer surface and the bulk flow in the pores are negligible because the diffusion coefficients in the gas phase are much higher than those in the polymer phase. The advection calculated by Eq. 9, which is a counter flow against the oxygen transport, blocks the oxygen supply and helps the water discharge from the pore in Eq. 10. The boundary conditions at the interface between the catalyst layer and the PEM are as follows

$$
\frac{d C_{O_{2}}^{g}}{d z}=0, \frac{d C_{H_{2} O}^{g}}{d z}=0, v=0 \quad \text { at } \quad z=0
$$

Here, the gradient of the vapor concentration was set to be 0 , which means that the water flux is 0 at the interface. This arises from an elimination of the effects of the water flux through the membrane depending on the PEM or anode structure and conditions in the anode, because the objective of this study is to investigate the effects of the cathode structure and properties on the cell performance. It is expected that the effect of the water flux through the membrane on the oxygen and vapor transports in the cathode pores is small as will be discussed with Fig. 3a, but a dry condition at the anode side decreases the proton conductivity of the polymer in the cathode catalyst layer. The water flux through the membrane must be considered in Eq. 11 under the low relative humidity conditions, for the accurate estimation of the conductivity of the cathode polymer. The concentrations of oxygen and vapor at the interface between the catalyst layer and the GDL were also set: $\mathrm{C}_{\mathrm{O}_{2}}^{I n}$ and $C_{\mathrm{H}_{2} \mathrm{O}}^{I n}$.

The oxygen and vapor fluxes, $\mathrm{N}_{\mathrm{O}_{2}}^{G D L}$ and $\mathrm{N}_{\mathrm{H}_{2} \mathrm{O}}^{G D L}$, were calculated by the following equations.

$$
\begin{aligned}
N_{O_{2}}^{G D L}=-D_{O_{2}}^{e f f, G D L} & \frac{1}{1+x_{O_{2}}} \cdot \frac{d C_{O_{2}}}{d z}=-D_{O_{2}}^{e f f, G D L} \frac{C^{g}}{\delta_{G D L}} \ln \left(\frac{C^{g}+C_{O_{2}}^{C h}}{C^{g}+C_{O_{2}}^{I n}}\right) \\
N_{\mathrm{H}_{2} \mathrm{O}}^{G D L} & =-D_{\mathrm{H}_{2} \mathrm{O}}^{e f f, G D L} \frac{1}{1-x_{\mathrm{H}_{2} \mathrm{O}} / 2} \cdot \frac{d C_{\mathrm{H}_{2} \mathrm{O}}}{d z} \\
& =D_{\mathrm{H}_{2} \mathrm{O}}^{e f f, G L} \frac{2 C^{g}}{\delta_{G D L}} \ln \left(\frac{C^{g}-C_{\mathrm{H}_{2} \mathrm{O}}^{C h}}{C^{g}-C_{\mathrm{H}_{2} \mathrm{O}}^{I n} / 2}\right)
\end{aligned}
$$

These were derived from equations similar to Eq. 10, where the rates of the oxygen consumption and vapor generation were zero in the GDL and the advection velocity, $v$, was given by the relationship, $\varepsilon_{C L} C^{g} v=i_{H+}(0) /(4 F)$, the integral of Eq. 9 over the whole thickness of the catalyst layer. In Eqs. 12 and 13, $D_{O_{2}}^{e f f, G D L}$ and
$D_{\mathrm{H}_{2} \mathrm{O}}^{e f f, G L}$ are the effective diffusion coefficients of the oxygen and water vapor, $\mathrm{C}_{\mathrm{O}_{2}}^{\mathrm{Ch}}$ and $\mathrm{C}_{\mathrm{H}_{2} \mathrm{O}}^{\mathrm{Ch}}$ are the concentrations of oxygen and vapor in the gas flow channel, and $\delta_{G D L}$ is the thickness of the GDL. Each of the fluxes was calculated using the condition that it equals the overall rates of oxygen consumption and water generation in the cathode catalyst layer. Each of the oxygen and vapor concentrations in the cathode gas flow channel was set to be a value intermediate between the inlet and the outlet gas compositions; the outlet gas composition was determined from the stoichiometric ratio of the cathode gas.

Effects of catalyst layer structure on effective properties.-In the catalyst layer, the effective proton conductivity of the polymer electrolyte and the effective diffusion coefficient in the pore of the catalyst layer depend on the catalyst layer structure. These properties, $\kappa^{\text {eff, } C L}$ and $D_{i}^{\text {eff, }, C L}$, were given using the Bruggeman correction factor as follows 14,16

$$
\begin{gathered}
\kappa^{e f f, C L}=\kappa\left[\left(1-\varepsilon_{C L}\right)\left\{1-\left(\frac{r_{a g}}{r_{a g}+\delta_{p}}\right)^{3}\right\}\right]^{1.5} \\
D_{i}^{e f f, C L}=D_{i}^{g} \frac{\varepsilon_{C L}}{\tau_{C L}}=D_{i}^{g} \varepsilon_{C L}^{1.5}
\end{gathered}
$$

Here, $\kappa$ is the proton conductivity of the polymer electrolyte, and $D_{i}^{g}$ is the diffusion coefficient of species $i$ in the catalyst layer pores. The Tomadakis and Sotirchos model, which is an equation to calculate the tortuosity of fibrous porous media, with the GDL porosity of 0.7 was used to calculate the effective diffusion coefficient in the GDL pores as follows ${ }^{16,17}$

$$
D_{i}^{e f f, G D L}=D_{M, i}^{g} \varepsilon_{G D L}\left(\frac{1-0.11}{\varepsilon_{G D L}-0.11}\right)^{-0.785}
$$

Here $D_{M, i}^{g}$ is the coefficient of molecular diffusion of species $i$.

Properties and standard conditions.-The proton conductivity of the polymer electrolyte, $\kappa$, was given by the following function of the water content in the polymer, $\lambda$, which depends on the water activity in the gas phase, $a\left(=C_{\mathrm{H}_{2} \mathrm{O}}^{g} / C_{\mathrm{H}_{2} \mathrm{O}}^{\text {sat }}\right) .{ }^{18}$

$$
\begin{array}{rc}
\kappa=100 \exp [1268(1 / 303-1 / T)](0.005139 \lambda-0.00326) \\
\lambda=0.043+17.81 a-39.85 a^{2}+36.0 a^{3} & (0 \leq a \leq 1) \\
\lambda=14+1.4(a-1) & (1 \leq a \leq 3)
\end{array}
$$

The diffusion coefficient in the catalyst layer pores, with the representative mean radius assumed to be $50 \mathrm{~nm}$, was calculated by the following equation in the transition region between molecular diffusion and Knudsen diffusion. ${ }^{12}$

$$
D_{i}^{g}=\left(1 / D_{M, i}^{g}+1 / D_{K, i}^{g}\right)^{-1}
$$

Here, $D_{K, i}^{g}\left(=2 / 3 \times\left(8 R T / \pi M_{i}\right)^{1 / 2} r_{C L}\right)$ is the coefficient of the Knudsen diffusion of species $i ; R$ is the universal gas constant, and $M_{i}$ is the molecular weight of a species $i$.

The standard conditions and parameters used in this study are provided in Table I. The conventionally used parameter set was applied here, although it has recently been pointed out that the actual structures have smaller agglomerate radiuses and thinner polymer films than the conventional values in Table I. ${ }^{15}$ To accommodate the new parameter set, a developed model considering the dissolution rate from the gas phase into the polymer is needed, and the effect of the resulting model differences on the cell performance will also be discussed with Fig. 6. The cathode gas in the inlet of the channel was set as air at $1 \mathrm{~atm}, 80^{\circ} \mathrm{C}$, and $80 \%$ relative humidity, and the stoichiometric ratio was 2.5 . The reference exchange current density, $i_{0}$, was based on the value in the literature $\left(i_{0}^{\text {ref }}=1.3874\right.$ 


\begin{tabular}{lc}
\hline $\begin{array}{l}\text { Table I. Simulation parameters and properties for the standard } \\
\text { condition here. }\end{array}$ \\
Parameter & Value \\
\hline Temperature, $T(\mathrm{~K})$ & 353.15 \\
Tafel slope, $b(\mathrm{~V})$ & $0.11589^{\mathrm{b}}$ \\
Reference exchange current density, $i_{0}\left(\mathrm{~A} \mathrm{~cm}^{-2}\right)$ & $1.6 \times 10^{-7}$ \\
Reference oxygen concentration, $C_{0, O_{2}}\left(\mathrm{~mol} \mathrm{~m}^{-3}\right)$ & 0.41 \\
Oxygen diffusion coefficient in polymer, $D_{O_{2}}\left(\mathrm{~m}^{2} \mathrm{~s}^{-1}\right)$ & $8.7 \times 10^{-10, \mathrm{~b}}$ \\
Oxygen concentration ratio at polymer-gas interface, $\gamma$ & $0.093^{\mathrm{c}, \mathrm{a}}$ \\
Platinum amount, $m_{P t}\left(\mathrm{mg} \mathrm{cm}{ }^{-2}\right)$ & 0.4 \\
Agglomerate radius, $r_{\mathrm{ag}}(\mathrm{nm})$ & 500 \\
Platinum particle radius, $r_{P t}(\mathrm{~nm})$ & 1.5 \\
Catalyst layer thickness, $\delta_{C L}(\mu \mathrm{m})$ & 10 \\
GDL thickness, $\delta_{G D L}(\mathrm{~mm})$ & 0.3 \\
Polymer thickness, $\delta_{p}(\mathrm{~nm})$ & 70 \\
Catalyst layer porosity, $\varepsilon_{C L}$ & 0.3 \\
GDL porosity, $\varepsilon_{G D L}$ & 0.7 \\
${ }^{\mathrm{a}}$ Reference 5 & \\
${ }^{\mathrm{b}}$ Reference 19 & \\
${ }^{\mathrm{c}}$ Reference 20 &
\end{tabular}

$\times 10^{-6} \mathrm{~A} \mathrm{~cm}^{-2}, C_{0, O}^{r e f}=4.43 \mathrm{~mol} \mathrm{~m}^{-3}$ ) (Ref. 19) using the relationship, $i_{0}=i_{0}^{r e f}\left(C_{0, O_{2}} / C_{0, O_{2}}^{r e f}\right)^{0.9}$, here $C_{0, O_{2}}$ is the oxygen concentration in the polymer in equilibrium with that in the cathode inlet gas. The ratio of the oxygen concentration in the polymer to that in the gas phase, $\gamma$, of 0.093 was used. ${ }^{5,20}$ In the one-dimensional simulation, $\delta_{C L} / 100$ was used as an adequate mesh size, $\Delta z$.

\section{Results and Discussion}

Effects of catalyst layer structure on cell performance.-The results of the simulation by the model for the standard condition are shown in Fig. 2. Figures $2 \mathrm{a}$ and $2 \mathrm{~b}$ are the distributions of the cathode reaction rate, $-d i_{H+} / d z$, and the cathode overpotential, $\eta$, in the catalyst layer, where the overpotentials, $\eta\left(\delta_{C L}\right)=-0.3,-0.5,-0.7$ $\mathrm{V}$, were set as the boundary conditions. Figure $2 \mathrm{a}$ shows that the reaction rate increases with decreasing cathode overpotential, this is because the absolute value of the overpotential contributes as a driving force for the cathode reaction in Eq. 6. The average reaction rate times the thickness of the catalyst layer, $\delta_{C L}$, corresponds to the cell current density and the higher reaction rate induces the higher cell current density. Simultaneously, the cathode overpotentials decrease near the PEM due to the proton conductivity resistance of the catalyst layer, and the amount of decrease in the overpotential becomes larger with higher reaction rates (Fig. 2b). However, the amount of the overpotential decrease is relatively small when considering the absolute value, and the distributions of the cathode reaction rates in Fig. 2a are approximately uniform across the thickness of the catalyst layer. In the later analysis, below, the polarization characteristics of the simulated results are evaluated by two values, the cell current density calculated from the reaction rate distribution and $\eta(0)$ at the interface between the catalyst layer and the PEM which corresponds to the total cathode overpotential.

Figure 3a shows these simulated polarization curves under the standard condition with only the porosity of the catalyst layer varied in the calculations. Here, the broken curve is for the result without advection, assuming that the advection velocity, $v$, is zero, under the standard condition. The effect of advection appears in the higher current density region, and the limiting current density where the cathode overpotential falls off to $-1.2 \mathrm{~V}$ is affected by the influence of advection. This happens because advection increases at high current densities; the increase in advection opposes the oxygen diffusion into the pores of the catalyst layer and here blocks the oxygen supply into the catalyst layer, but the effect is small as shown in Fig. 3a, where the curves with and without the advection are not very different. The effect of advection on the oxygen supply in the GDL is expressed in Eq. 12 by the factor, $1 /\left(1+x_{O_{2}}\right)$. In evaluating this factor, it must be remembered that the advection effect becomes larger in conditions with high oxygen concentrations like in operation with pure oxygen as the cathode gas.

The limiting current density due to the concentration overpotential decreases as the porosity of the catalyst layer increases in Fig. 3a, and to investigate details of the effect of the concentration overpotential, the distribution of oxygen concentrations is shown in Fig. $3 \mathrm{~b}$ for each of the three catalyst layer porosities at $I=2.0 \mathrm{~A}$ $\mathrm{cm}^{-2}$. The oxygen concentrations are the values of the concentrations of oxygen dissolved in the polymer, and the solid and broken lines are for at the gas-polymer and the polymer-agglomerate interfaces respectively. The oxygen concentrations in the catalyst layer pores at the interface between the catalyst layer and the GDL, $\mathrm{C}_{\mathrm{O}_{2}}^{\mathrm{In}}$, are determined by the mass transport resistance of the GDL and the consumption rate depending on $I$ in the entire catalyst layer, here the oxygen concentrations in the polymer on the surface (solid lines) at the interface with the GDL $\left(z / \delta_{C L}=1\right), \gamma C_{O_{2}}^{I n}$, have the same value at $I=2.0 \mathrm{~A} \mathrm{~cm}^{-2}$ in Fig. $3 \mathrm{~b}$. These concentrations decrease slightly approaching the interface with the PEM $\left(z / \delta_{C L}=0\right)$, and the amount of the decrease increases with lower porosities of the catalyst layer, as the effective oxygen diffusion coefficient decreases with decreasing catalyst layer porosity, as suggested by Eq. 15 . Additionally, the oxygen dissolved in the polymer transports to the interface with the carbon agglomerate, which is the cathode reaction site. The concentration difference between the interfaces of the catalyst layer pores and the carbon agglomerate (the distance between the solid and broken lines in Fig. 3b) is caused by the oxygen diffusion resistance of the polymer, and the difference increases with higher catalyst layer porosities. This can be understood by the changes in the agglomerate numbers, increasing the catalyst layer porosity with a constant thickness reduces the number of agglomerates in the catalyst layer and so increases the oxygen consumption rate per agglomerate, which in turn induces an increase in the effect of the diffusion resistance in the polymer. As a result, the higher catalyst layer porosity decreases the oxygen concentration at the reaction site despite the higher oxygen concentration in the gas phase
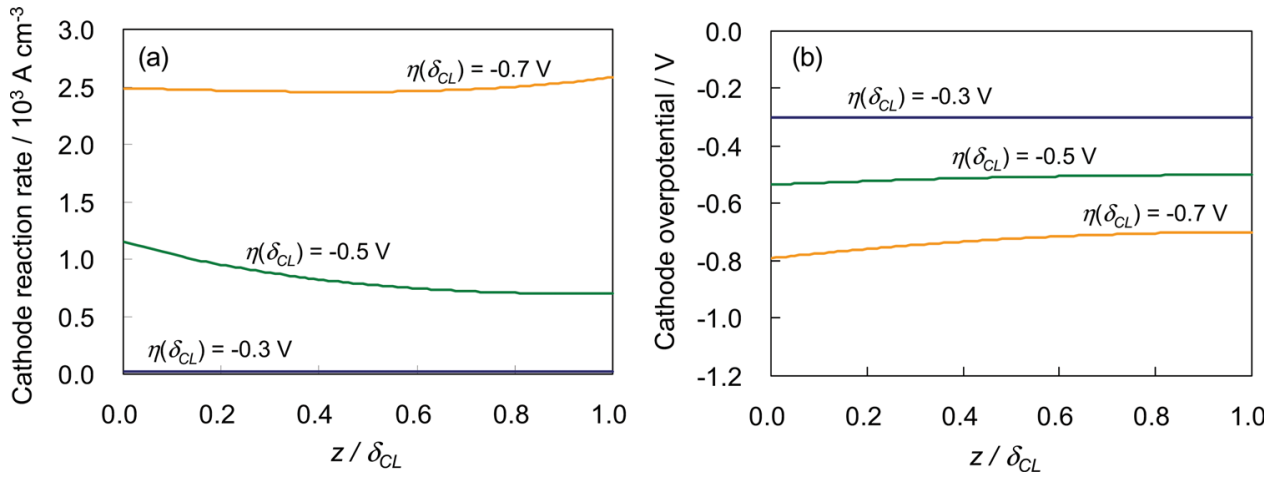

Figure 2. (Color online) (a) Cathode reaction rate and (b) cathode overpotential in the catalyst layer under the standard conditions in Table I for three different boundary conditions of $\eta\left(\delta_{C L}\right)=-0.3$, $-0.5,-0.7 \mathrm{~V}$. 

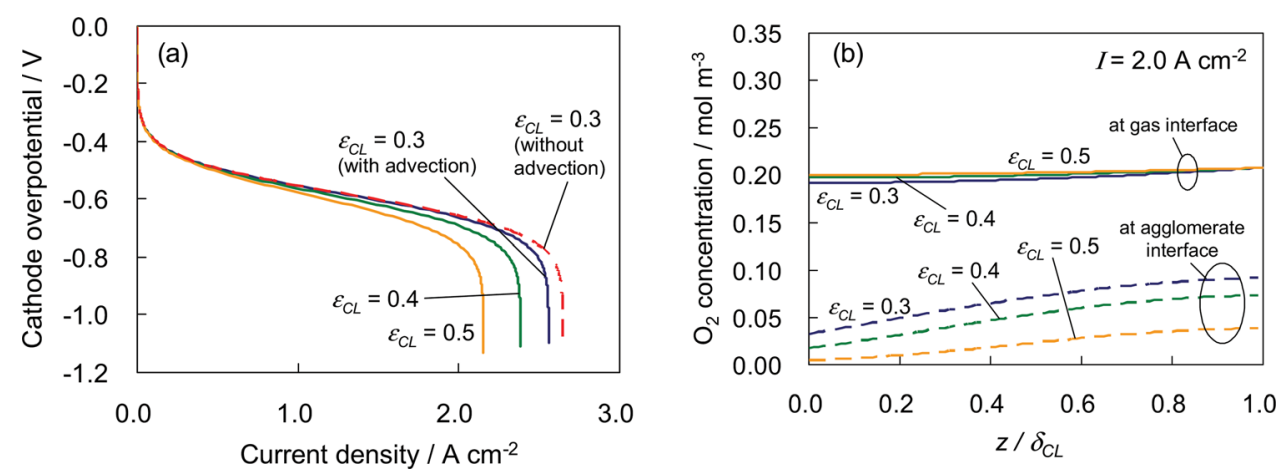

Figure 3. (Color online) Effect of the porosity of the catalyst layer, $\varepsilon_{C L}$ : (a) polarization curves and (b) distribution of oxygen concentrations in the polymer in the catalyst layer for $\varepsilon_{C L}=0.3,0.4,0.5$. The other parameters are the same as those at the standard condition. The broken polarization curve is for the result without advection, assuming that the advection velocity, $v$, is zero, under the standard conditions. and it increases the effect of the concentration overpotential. Overall, the effect of the catalyst layer porosity change on the oxygen diffusion resistance in the polymer plays a dominant role in the cell performance. Theses results are consistent with the recently reported measurements that the oxygen permeation through the polymer film is a major portion of the total transport resistance and that the contribution from the diffusion resistance in the pores of the catalyst layer is much smaller than the contribution of the other parameters contributing to the total resistance. ${ }^{21}$ However, it must be borne in mind that the higher current density with the lower catalyst layer porosity may increase the effect of a liquid water film on the oxygen transport in the pore and that an accurate assessment of the water film formation is needed under high relative humidity and high current density conditions.

Figures 4 and 5 show the results of the effects of the polymer electrolyte thickness, $\delta_{p}$, and the catalyst layer thickness, $\delta_{C L}$, which are estimated to have a strong influence on the oxygen diffusion resistance in the polymer. In the polarization curves of Figs. 4a and 5, the limiting current density increases significantly with decreasing polymer thickness and increasing catalyst layer thickness. This is because the oxygen diffusion resistance decreases with decreasing polymer thickness, and the agglomerate number increases and the oxygen consumption rate per agglomerate decreases with increasing catalyst layer thickness. However, the cathode overpotential decreases even in the low current density region with the $18 \mathrm{~nm}$ thick polymer and with the $40 \mu \mathrm{m}$ thick catalyst layer. The distribution of the cathode reaction rate in the catalyst layer at $I=0.5 \mathrm{~A} \mathrm{~cm}^{-2}$ is shown in Fig. $4 \mathrm{~b}$. The reaction rates are nearly constant with the 140 and $70 \mathrm{~nm}$ thick polymers, but there is a considerably large variation in the reaction rates with the $18 \mathrm{~nm}$ thick polymer. With the $18 \mathrm{~nm}$ thick polymer, the reaction is active near the PEM and there is little reaction near the GDL. This is because the effective proton conductivity decreases with decreases in the polymer thickness as in Eq. 14, and the distribution of the cathode overpotential like in Fig. $2 b$ becomes larger. The deterioration in the performance of the $40 \mu \mathrm{m}$ thick catalyst layer is also considered to be caused by similar reasons (Fig. 5). Thus, the thinner polymer electrolyte and the thicker catalyst layer are effective to increase the limiting current density, but it is important to ensure sufficient proton conductivity to maintain the overpotential practically constant in the catalyst layer. It must be noted that an inadequate proton conductivity induces performance deteriorations even in the low current density region.

Figure 6a shows the results of the effects of the polymer electrolyte thickness, $\delta_{p}$, and Fig. $6 \mathrm{~b}$ shows those of the catalyst layer thickness, $\delta_{C L}$, using a developed model to investigate the effect of the conventional model in this study on the dependence of these parameters. The agglomerate radius was changed to $29 \mathrm{~nm}$ and the polymer thickness was varied from 1.4 to $11.2 \mathrm{~nm} .{ }^{15}$ The other parameters were same as those at the standard condition in Table I. Additionally, the effect of the oxygen dissolution rate from the gas phase into the polymer was introduced: the dissolution resistance term, $r_{a g}^{2} /\left[\left(r_{a g}+\delta_{p}\right)^{2} k_{\text {diss }}\right]$, was added to the bracket on the right hand side in Eq. 5; here, the dissolution rate constant, $k_{\text {diss }}=8 \times 10^{-4} \mathrm{~m} \mathrm{~s}^{-1}$, was used. ${ }^{15}$ The changes in the effect of the polymer thickness in Fig. 6a are similar to the results in Fig. $4 \mathrm{a}$, but the contribution of the polymer thickness becomes smaller. This is because the effect of the polymer thickness on the dissolution resistance is smaller than the effect on the diffusion resistance in the polymer (the first term in the bracket on the right hand side of Eq. 5). The very thick polymer film in the conventional model can be considered to stand for the effective polymer thickness including the oxygen dissolution resistance, as pointed out by Nonoyama et al. ${ }^{21}$ The contribution of the catalyst layer thickness in the polarization curve by the model proposed here with a $2.8 \mathrm{~nm}$ thick polymer film in Fig. $6 \mathrm{~b}$ was very similar to that by the conventional model in Fig. 5. The results show that with increasing catalyst layer thickness, the increase in the number of agglomerates and the decrease in the oxygen consumption rate per agglomerate affect the decrease in the oxygen concentration by the dissolution resistance similarly. Thus, the conventional model can simulate the effect of the variation in this parameter but tends to overestimate the effect of the polymer thickness (the effective thickness including the oxygen diffusion and dissolution resistances) on the cell performance.

Equations to evaluate structure effects and optimization.-As detailed in the analysis of the previous section, the catalyst layer structure affects cell performance in complicated ways through changes in the oxygen diffusion resistance in the polymer electrolyte, the proton conductivity in the catalyst layer, and the oxygen transport
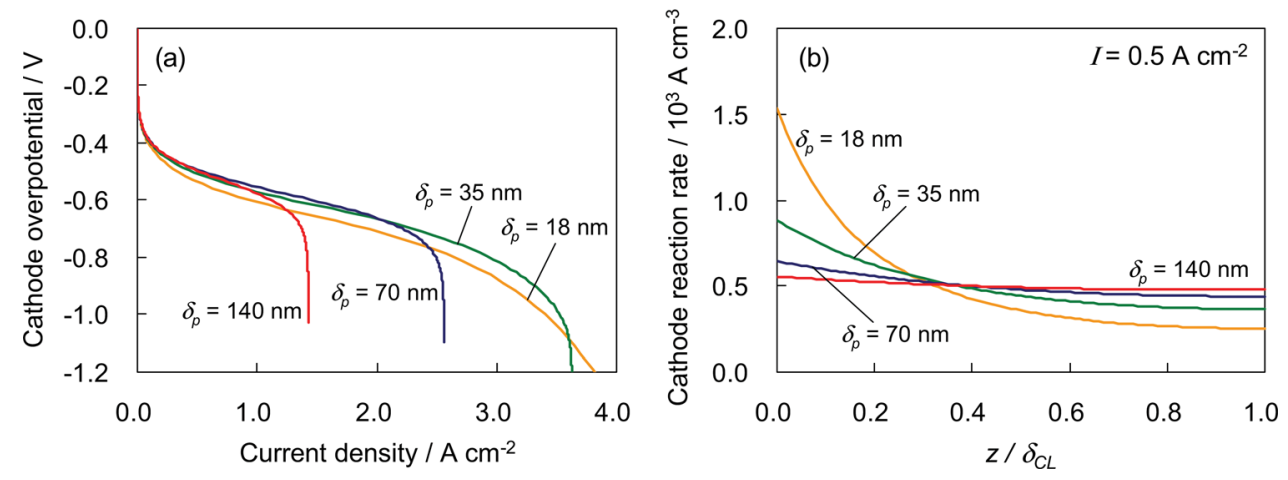

Figure 4. (Color online) Effect of the polymer thickness around the agglomerate, $\delta_{p}$ : (a) polarization curves and (b) distribution of cathode reaction rates in the catalyst layer for $\delta_{p}=18,35,70,140 \mathrm{~nm}$. The other parameters are the same as those at the standard condition. 


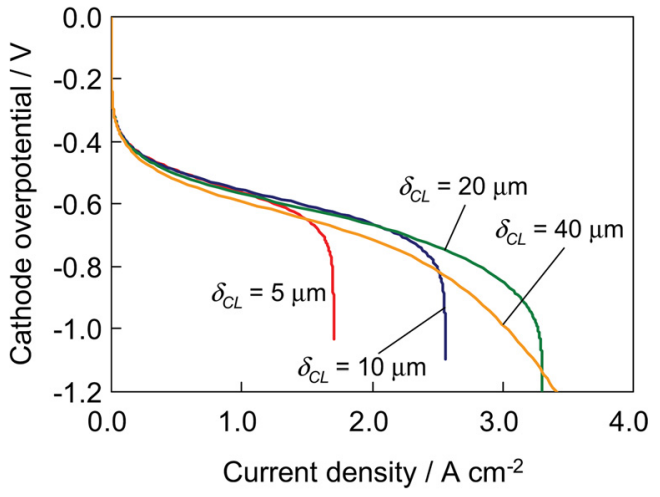

Figure 5. (Color online) Effect of the catalyst layer thicknesses, $\delta_{C L}$ : polarization curves for $\delta_{C L}=5,10,20.40 \mu \mathrm{m}$. The other parameters are the same as those at the standard condition.

resistance in the pores of the catalyst layer. This section develops equations to evaluate and understand the effects of the catalyst layer structure and properties on the cell performance and confirms the applicability of the equations with the model simulation.

To examine the performance deterioration due to the inadequate proton conductivity in the low current density region, the results of changing the porosity of the catalyst layer from 0.3 to 0.5 , the polymer thickness from 18 to $140 \mathrm{~nm}$, and the catalyst layer thickness from 5 to $40 \mu \mathrm{m}$ are shown in Fig. 7. The abscissa is the parameter representing the electric resistance within the catalyst layer, $\delta_{C L} / \kappa^{\text {eff, } C L}$, and the ordinate is the cathode overpotential, $\eta(0)$, at $0.5 \mathrm{~A} \mathrm{~cm}^{-2}$. The proton conductivity of the polymer, $\kappa$, is given by a constant value for the inlet gas composition, and the results where the limiting current density is less than $1.0 \mathrm{~A} \mathrm{~cm}^{-2}$ are disregarded because of the too large effect of the concentration overpotential at these overpotential values. Fig. 7 shows that the data generally fall on a curve and that the parameter of the abscissa provides a measure of the deterioration due to the low proton conductivity in the low current density region. The cathode overpotential at $0.5 \mathrm{~A} \mathrm{~cm}^{-2}$ is maintained approximately constant where $\delta_{C L} / \kappa^{\text {eff, } C L}$ is below $200 \mathrm{~m} \Omega \mathrm{cm}^{2}$, and is lower above $200 \mathrm{~m} \Omega$ $\mathrm{cm}^{2}$ (In Fig. 7, the broken line represents this threshold, $200 \mathrm{~m} \Omega \mathrm{cm}^{2}$.) The results in Fig. 7 suggest that the catalyst layer structure must satisfy the following relationship to prevent performance deterioration due to low current densities.

$$
\delta_{C L} / \kappa^{e f f, C L} \leq 200 \mathrm{~m} \Omega \mathrm{cm}^{2}
$$

The threshold value is within the range of ac impedance measurement results, $100-240 \mathrm{~m} \Omega \mathrm{cm}^{2}{ }^{22}$

Now a further equation to evaluate the limiting current density determined by the mass transfer alone, is developed using the following assumptions based on the results in the previous section:

(a) the effect of advection in the catalyst layer and in the GDL is negligible, (b) the oxygen concentration at the surface of the carbon agglomerates (reaction sites) at the interface between the catalyst layer and the PEM is zero at the limiting current density,

(c) the oxygen concentration distribution in the catalyst layer pores is approximated by a quadratic function of $z$ because the distribution of the reaction is uniform in the catalyst layer.

Using these assumptions, the limiting diffusion current density, $I_{L D}$, can be expressed by the following equation (for further details refer to the Appendix).

$$
I_{L D}=4 F C_{O_{2}}^{C h}\left[\frac{1}{n_{a g} \delta_{C L}} \cdot \frac{1}{4 \pi r_{a g}\left(r_{a g}+\delta_{p}\right) \gamma} \cdot \frac{\delta_{p}}{D_{O_{2}}^{p}}+\frac{\delta_{C L}}{3 D_{O_{2}}^{e f f, C L}}+\frac{\delta_{G D L}}{D_{O_{2}}^{e f f, G D L}}\right]^{-1}
$$

In the bracket on the right hand side of Eq. 20, the first, the second, and the third terms represent the oxygen diffusion resistance in the polymer electrolyte, in the catalyst layer pores, and in the GDL respectively. The $n_{a g} \delta_{C L}$ in the first of these terms represents the number of agglomerates per unit area of catalyst layer, and the equation considers that the oxygen diffusion resistance is inversely proportional to the number of agglomerates. Further, Eq. 20 can be transformed into Eq. 21, where $n_{a g}$ and $D_{O_{2}}^{e f f}, C L$ are expressed by the relationship with the catalyst layer structure.

$$
I_{L D}=4 F C_{O_{2}}^{C h}\left[\frac{\left(r_{a g}+\delta_{p}\right)^{2}}{3\left(1-\varepsilon_{C L}\right) \delta_{C L} r_{a g} \gamma} \cdot \frac{\delta_{p}}{D_{O_{2}}^{p}}+\frac{1}{3 \varepsilon_{C L}^{1.5}} \cdot \frac{\delta_{C L}}{D_{O_{2}}^{g}}+\frac{\delta_{G D L}}{D_{O_{2}}^{e f f, G D L}}\right]^{-1}
$$

Equation 21 explains, quantitatively, that the limiting current density increases with decreasing polymer thickness, $\delta_{p}$, and increasing catalyst layer thickness, $\delta_{C L}$, as in Figs. $4 \mathrm{a}$ and 5, because the oxygen diffusion resistance in the polymer electrolyte (the first term in the right side bracket of Eq. 21) decreases. With decreasing catalyst layer porosity, the oxygen diffusion resistance in the catalyst layer pores (the second term in the bracket) increases but the diffusion resistance in the polymer decreases. The effect of the decrease in the resistance in the polymer is dominant under the conditions used in this study, as shown in Fig. 3, and it can be evaluated by Eq. 21. Equation 21 can also be used to evaluate the measurement results that the diffusion resistance in the catalyst layer pores is much smaller than the other resistances. ${ }^{21}$ Figure 8 is a plot of the limiting current densities calculated by Eq. 21 (abscissa) and the agglomerate model (ordinate) for the various structures like in the analysis investigating the effect of the proton conductivity in Fig. 7 (the porosity of the catalyst layer from 0.3 to 0.5 , the polymer thickness from 18 to $140 \mathrm{~nm}$ and the catalyst layer thickness from 5 to 40 $\mu \mathrm{m})$. The straight line in Fig. 8 shows the case where the value calculated by Eq. 21 is the same as that by the agglomerate model simulation. The filled diamonds are for the conditions satisfying Eq. 19, and the open diamonds for those not satisfying Eq. 19 $\left(\delta_{C L} / \kappa^{\text {eff }, C L}>200 \mathrm{~m} \Omega \mathrm{cm}^{2}\right)$. The filled diamonds plot very nearly
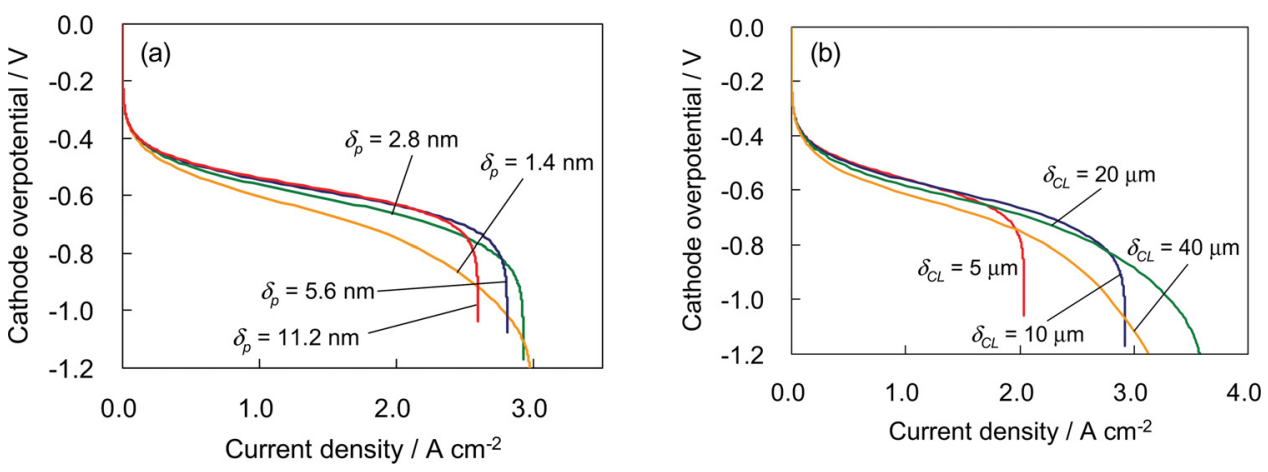

Figure 6. (Color online) Effects of the polymer thickness, $\delta_{p}$, and the catalyst layer thicknesses, $\delta_{C L}$, by the developed model. (a) Polarization curves for $\delta_{p}=1.4$, $2.8,5.6,11.2 \mathrm{~nm}$; the agglomerate radius, $r_{a g}=29 \mathrm{~nm}$ and the other parameters are the same as those at the standard conditions. (b) Polarization curves for $\delta_{C L}=5$, $10,20.40 \mu \mathrm{m}$; the agglomerate radius, $r_{a g}=29 \mathrm{~nm}$, the polymer thickness, $\delta_{p}=2.8 \mathrm{~nm}$, and other parameters same as those at the standard condition. 


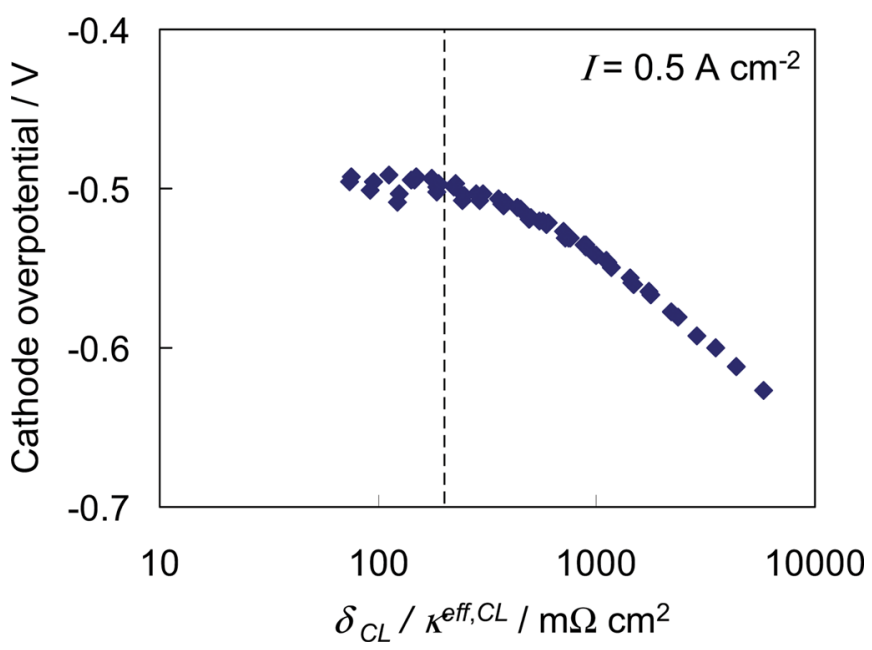

Figure 7. (Color online) Effect of proton conductivity on decreases in the cathode overpotential: the abscissa is the parameter representing the electric resistance within the catalyst layer, $\delta_{C L} / \kappa^{e f f, C L}$, and the ordinate is the cathode overpotential, $\eta(0)$, at $I=0.5 \mathrm{~A} \mathrm{~cm}^{-2}$. The porosity of the catalyst layer from 0.3 to 0.5 , the polymer thickness from 18 to $140 \mathrm{~nm}$ and the catalyst layer thickness from 5 to $40 \mu \mathrm{m}$. The broken line represents the threshold, $200 \mathrm{~m} \Omega \mathrm{cm}^{2}$ in Eq. 19.

on the straight line, and this shows that Eq. 21 can estimate the limiting current density well in the conditions satisfying Eq. 19. The reason why the open diamonds plot away from the straight line is that power generation becomes impossible due to insufficient proton conductivity before the current density reaches the current density limited by diffusion in the model simulation. Thus, it can be concluded that Eq. 21 can be applied to discuss the effects of various parameters on the limiting current density, in the conditions satisfying Eq. 19.

The equations to evaluate the proton conductivity, Eq. 19, and the limiting diffusion current density, Eq. 21, developed here make it possible to optimize the catalyst layer structure in a straightforward manner. In the conditions used in this study, a lower porosity in the catalyst layer is advantageous, because the porosity has a stronger influence on the oxygen diffusion resistance in the polymer

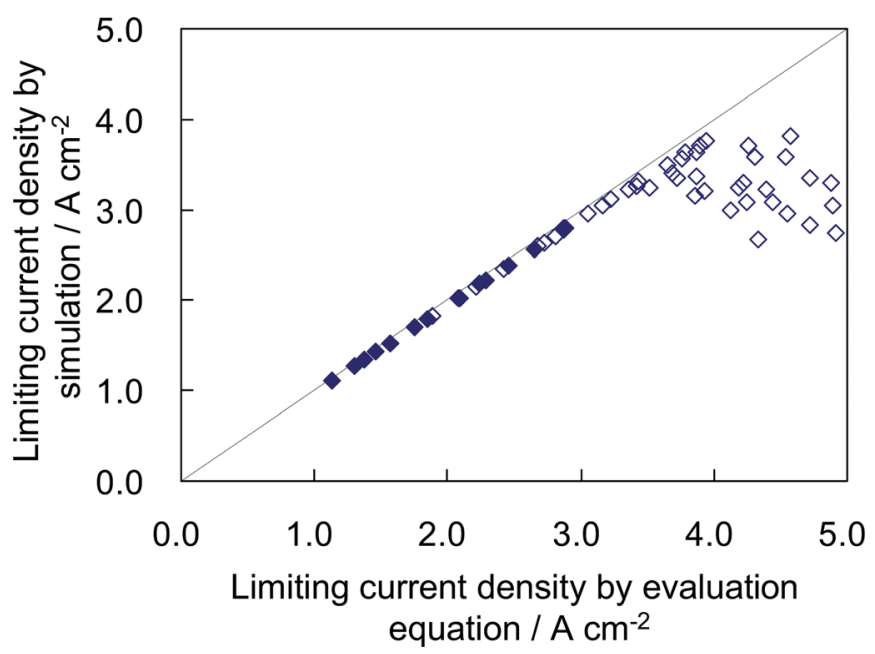

Figure 8. (Color online) Plot of the limiting current density by simulation vs. that by Eq. 21 . The porosity of the catalyst layer from 0.3 to 0.5 , the polymer thickness from 18 to $140 \mathrm{~nm}$ and the catalyst layer thickness from 5 to $40 \mu \mathrm{m}$. The straight line shows the case where the value calculated by Eq. 21 is the same as that by the agglomerate model simulation. The filled diamonds are for the conditions satisfying Eq. 19, and the open diamonds for those not satisfying Eq. 19. than on that in the catalyst layer pores, as expressed in Eq. 21. Therefore, the catalyst layer porosity was set to be 0.3 as a minimum value for the optimum structure in the following. Figure 9 shows the limiting current density and the catalyst layer thickness vs. the thickness of the polymer, with the catalyst layer thickness selected to be the maximum value satisfying Eq. 19 to ensure adequate proton conductivity and to achieve a maximum limiting current density. A thinner polymer electrolyte is effective to increase the limiting current density, but at the same time a thinner catalyst layer thickness is needed to ensure sufficient proton conductivity, which then decreases the limiting current density. The structure of the model suggests an optimum parameter setting where the advantages of the thin polymer thickness and the disadvantages of the thin catalyst layer thickness are dominant, at polymer thicknesses above and below about $40 \mathrm{~nm}$ respectively. In the conditions used in this study, there is a maximum limiting current density, around $3.1 \mathrm{~A} \mathrm{~cm}^{-2}$, for the catalyst layer structure of $\varepsilon_{C L}=0.3, \delta_{p}=45 \mathrm{~nm}, \delta_{C L}=7.8 \mu \mathrm{m}$. Thus, it is possible to investigate the dominating parameters and evaluate the structure of the catalyst layer for a range of conditions using the evaluation equations proposed here.

Parameters for reducing platinum use.-This section discusses effective measures for reductions in platinum usage. Figure 10 shows a comparison of the influences between the structure optimization suggested above and increase in the platinum amount, $m_{P t}$. The influence of the increase in the ratio of oxygen concentration in the polymer electrolyte to that in the gas phase at the polymer-gas interface, $\gamma$, which will be discussed later, are also shown in Fig. 10. In the optimum structure case, the optimum value set $\left(\varepsilon_{C L}=0.3\right.$, $\delta_{p}=45 \mathrm{~nm}, \delta_{C L}=7.8 \mu \mathrm{m}$ ) was applied to the simulation and the other parameters are those at the standard condition, and in the case with twice the amount of platinum, only $m_{P t}$ was changed and then only the number density of platinum particles, $n_{P t}$, was changed from the standard condition. In Fig. 10, the amount of platinum affects the cell performance in the low and medium current density regions, this result arises as larger amounts of platinum catalyst reduces the activation overpotential. By contrast, the optimization of the catalyst layer structure is effective to improve the cell performance in the high current density region, here the effect of the optimization on the change in the electrochemically active platinum surface area was not considered in the model. As a method to decrease the oxygen transport resistance in the polymer electrolyte surrounding the agglomerate, attention is on the increase in the oxygen concentration ratio, $\gamma$, and its effect is also shown in Fig. 10. The broken curve is calculated under the condition that only $\gamma$ was increased by $50 \%$ from the standard condition. Increase in the

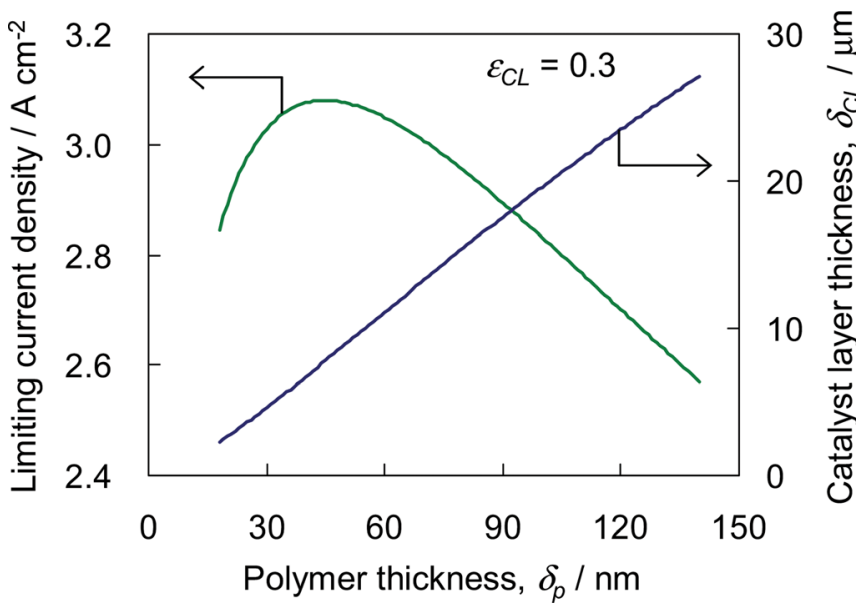

Figure 9. (Color online) Catalyst layer thickness for the equality condition in Eq. 19 (right ordinate) and limiting current density calculated by Eq. 21 (left ordinate) plotted against polymer thickness. The porosity of the catalyst layer is fixed at 0.3 . 


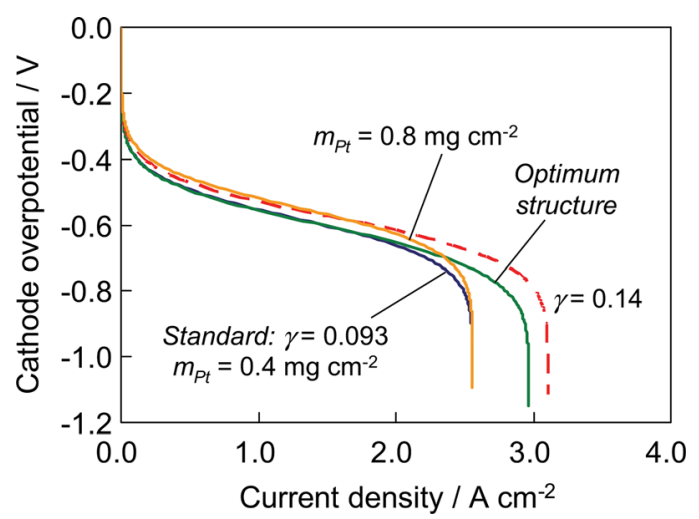

Figure 10. (Color online) Effects of the structure optimization, the platinum amount, $m_{P t}$, and the ratio of oxygen concentration in the polymer electrolyte to in the gas phase at the polymer-gas interface, $\gamma$. The optimum structure case: $\varepsilon_{C L}=0.3, \delta_{p}=45 \mathrm{~nm}, \delta_{C L}=7.8 \mu \mathrm{m}$ (green curve). With the twice platinum amount case: $m_{P t}=0.8 \mathrm{mg} \mathrm{cm}{ }^{-2}$ (yellow curve) the number density of platinum particles, $n_{P t}$, also becomes twice. In the higher oxygen concentration ratio case (broken red line): $\gamma=0.14, \mathrm{C}_{0, O_{2}}$ and $i_{0}$ are also changed. The other parameters are the same as those at the standard condition (blue curve).

concentration ratio, $\gamma$, reduces both the concentration overpotential in the high current density region as well as the activation overpotential at low and medium current densities. The improvement of the activation overpotential is caused by the increase in the reference exchange current density, $i_{0}$, due to the increase in the oxygen concentration dissolved in the polymer in equilibrium with that in the cathode inlet gas, $C_{0, O_{2}}$. Although it is difficult to control the concentration ratio, $\gamma$, the contribution of $\gamma$ to the cell performance is significantly larger. This means that a lower concentration of dissolved oxygen in the polymer electrolyte has a very large effect on the cathode reaction of the PEFC. This result suggests the possibility that increasing the oxygen concentration ratio, phrased differently, increasing the oxygen solubility in the polymer, would be effective to allow large reductions in platinum usage.

\section{Conclusions}

Using an agglomerate model of the cathode catalyst layer in a PEFC, factors dominating the cell performance and strategies for reducing platinum use were investigated. The major conclusions may be summarized as follows:

1. The oxygen transport resistance through the polymer electrolyte covering the carbon agglomerate has a dominating effect on the cathode polarization characteristics under the standard conditions of this study. To reduce the decrease in oxygen concentration due to the transport through the polymer, it is effective to reduce the thickness of the polymer and to decrease the oxygen diffusion rate per carbon agglomerate with a thicker catalyst layer.

2. A higher porosity of the catalyst layer, a thinner polymer electrolyte around the carbon agglomerate, and a thicker catalyst layer decreases the effective proton conductivity in the catalyst layer. The resulting inadequate proton conductivity causes a nonuniform distribution of the cathode overpotential and induces a significant performance deterioration even at low current densities.

3. Equations of evaluation were developed to enable a straightforward method of estimating the effects of the cathode catalyst layer structure and properties on the cell performance, and the effectiveness of the proposed equations was confirmed by a comparison of the results calculated by the equations and by model simulation. The equations of evaluation developed here make it possible to understand the influence of the various factors and to optimize the structure of cathode catalyst layer simply.
4. Increases in the oxygen solubility in the polymer electrolyte of the catalyst layer results in significant improvements in both the concentration overpotential in the high current density region as well as the activation overpotential at low and medium current densities. Overall, increasing the oxygen solubility of the polymer is highly effective to achieve a large reduction of platinum usage in PEFCs.

\section{Appendix}

Assuming that the oxygen concentration at the surface of the carbon agglomerate, $\mathrm{C}_{\mathrm{O}_{2}}^{*}$, is zero at the interface between the PEM and the catalyst layer, the oxygen diffusion flux at the agglomerate surface in the radial direction, $J_{O_{2}}^{a g}$, can be expressed by the following equation using Eq. 2 with the oxygen concentration in the gas phase at the polymer surface, $C_{O_{2}}^{g}$

$$
J_{O_{2}}^{a g}=-C_{O_{2}}^{g} D_{O_{2}}^{p} \frac{\left(r_{a g}+\delta_{p}\right) \gamma}{\delta_{p} r_{a g}}
$$

Because the effect of advection in the catalyst layer is negligible, the entire oxygen diffusion flux at the interface between the catalyst layer and the GDL in the $z$ direction, $J_{O_{2}}^{C L}$, is expressed by the following equation

$$
J_{O_{2}}^{C L}=-4 \pi r_{a g}\left(r_{a g}+\delta_{p}\right) \gamma \frac{D_{O_{2}}^{p}}{\delta_{p}} n_{a g} \int_{0}^{\delta_{C L}} C_{O_{2}}^{g} d z
$$

Approximating the $z$ direction distribution of the oxygen concentration in the pores of the catalyst layer by a quadratic function and using Eq. A-3 as the boundary condition, Eq. A-2 transforms into Eq. A-4

$$
\begin{array}{ll}
d C_{O_{2}}^{g} / d z=0 & \text { at } \quad z=0 \\
C_{O_{2}}^{g}=C_{O_{2}}^{I n}, \quad d C_{O_{2}}^{g} / d z=-J_{O_{2}}^{C L} / D_{O_{2}}^{e f f, C L} & \text { at } \quad z=\delta_{C L} \\
J_{O_{2}}^{C L}=C_{O_{2}}^{I n}\left[\frac{1}{n_{a g} \delta_{C L}} \cdot \frac{1}{4 \pi r_{a g}\left(r_{a g}+\delta_{p}\right) \gamma} \cdot \frac{\delta_{p}}{D_{O_{2}}^{p}}+\frac{\delta_{C L}}{3 D_{O_{2}}^{e f f, C L}}\right]^{-1}
\end{array}
$$

In the GDL, the oxygen diffusion flux at the interface between the catalyst layer and the $\mathrm{GDL}$ in the $z$ direction, $J_{\mathrm{O}_{2}}^{G D L}$, is expressed by the following equation

$$
J_{O_{2}}^{G D L}=-D_{O_{2}}^{e f f, G D L} \frac{C_{O_{2}}^{C h}-C_{O_{2}}^{I n}}{\delta_{G D L}}
$$

Using $J_{O_{2}}^{C L}=J_{O_{2}}^{G D L}$ and eliminating $C_{O_{2}}^{I n}$ from Eqs. A-4 and A-5 yields the following equation.

$$
J_{O_{2}}^{C L}=J_{O_{2}}^{G D L}=-C_{O_{2}}^{C h}\left[\frac{1}{n_{a g} \delta_{C L}} \cdot \frac{1}{4 \pi r_{a g}\left(r_{a g}+\delta_{p}\right) \gamma} \cdot \frac{\delta_{p}}{D_{O_{2}}^{p}}+\frac{\delta_{C L}}{3 D_{O_{2}}^{e f f, C L}}+\frac{\delta_{G D L}}{D_{O_{2}}^{e f f, G D L}}\right]^{-1}
$$

Multiplying Eq. A- 6 by $4 F$ gives Eq. 20 as the equation for the limiting diffusion current density, $I_{L D}$

\section{List of Symbols}

A area ratio of the reaction site to the carbon agglomerate surfaces

a water activity

$b$ Tafel slope, $\mathrm{V}$

$C$ molar concentraition, $\mathrm{mol} \mathrm{m}^{-3}$

$C_{0}$ reference molar concentration, $\mathrm{mol} \mathrm{m}^{-3}$

$D$ diffusion coefficient, $\mathrm{m}^{2} \mathrm{~s}^{-1}$

$D_{K} \quad$ Knudsen diffusion coefficient, $\mathrm{m}^{2} \mathrm{~s}^{-1}$

$D_{M}$ molecular diffusion coefficient, $\mathrm{m}^{2} \mathrm{~s}^{-1}$

$F \quad$ Faraday's constant, $\mathrm{C} \mathrm{mol}^{-1}$

$I$ current density, $\mathrm{A} \mathrm{m}^{-2}$

$I_{L D}$ limiting diffusion current density, $\mathrm{A} \mathrm{m}^{-2}$

$i_{H+}$ proton current density, $\mathrm{A} \mathrm{m}^{-2}$

$i_{0}$ reference exchange current density, $\mathrm{A} \mathrm{m}^{-2}$

$J$ molar diffusion flux, $\mathrm{mol} \mathrm{m} \mathrm{m}^{-2} \mathrm{~s}^{-1}$

$k_{d i s s}$ oxygen dissolution rate constant from the gas to the polymer, $\mathrm{m} \mathrm{s}^{-1}$

$k_{\text {ORR }}$ reaction constant of the oxygen reduction reaction, $\mathrm{m} \mathrm{s}^{-1}$

$M$ molecular weight, $\mathrm{kg} \mathrm{mol}^{-1}$

$m$ supported mass per unit area, $\mathrm{kg} \mathrm{m}^{-2}$ 
$N$ molar flux, $\operatorname{mol~m}^{-2} \mathrm{~s}^{-1}$

$n$ number density, $\mathrm{m}^{-3}$

$R$ universal gas constant, $\mathrm{J} \mathrm{K}^{-1} \mathrm{~mol}^{-1}$

$r$ radius, $\mathrm{m}$

$S$ surface area, $\mathrm{m}^{2}$

$T$ temperature, $\mathrm{K}$

$v$ advection velocity, $\mathrm{m} \mathrm{s}^{-1}$

$x$ mole fraction

$z$ coordinate in the catalyst layer, $\mathrm{m}$

Greek

$\gamma$ equilibrium ratio of oxygen concentrations in the polymer to in the gas phase

$\delta$ thickness, $\mathrm{m}$

$\varepsilon$ porosity

$\eta$ cathode overpotential, $\mathrm{V}$

$\kappa$ proton conductivity, $\mathrm{S} \mathrm{m}^{-1}$

$\lambda$ water content of the polymer

$\rho$ density, $\mathrm{kg} \mathrm{m}^{-3}$

$\tau$ tortuosity

$\dot{\omega}$ rate of generation, $\mathrm{mol} \mathrm{m}^{-3} \mathrm{~s}^{-1}$

Subscripts and supersctipts

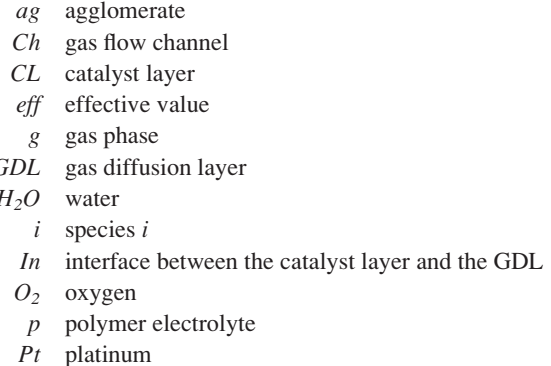

ref reference condition

$S$ polymer at the interface of the gas phase

sat saturated state

* polymer at the interface of the carbon agglomerate

\section{References}

1. D. Song, Q. Wang, Z. Liu, M. Eikerling, Z. Xie, T. Navessin, and S. Holdcroft, Electrochim. Acta, 50, 3347 (2005)

2. M. Secanell, B. Carnes, A. Suleman, and N. Djilali, Electrochim. Acta, 52, 2668 (2007).

3. M. Eikerling, J. Electrochem. Soc., 153, E58 (2006).

4. F. Jaouen, G. Lindbergh, and G. Sundholm, J. Electrochem. Soc., 149, A437 (2002)

5. W. Sun, B. A. Peppley, and K. Karan, Electrochim. Acta, 50, 3359 (2005).

6. M. Secanell, K. Karan, A. Suleman, and N. Dijlali, Electrochim. Acta, 52, 6318 (2007).

7. K. Karan, Electrochem. Commun., 9, 747 (2007).

8. S. Kamarajugadda and S. Mazumder, J. Power Sources, 183, 629 (2008).

9. G. Lin, W. He, and T. V. Nguyen, J. Electrochem. Soc., 151, A1999 (2004).

10. C. Y. Du, X. Q. Cheng, T. Yang, G. P. Yin, and P. F. Shi, Electrochem. Commun., 7, 1411 (2005).

11. M. Chisaka and H. Daiguji, Electrochem. Commun., 8, 1304 (2006).

12. P. P. Mukherjee and C.-Y. Wang, J. Electrochem. Soc., 153, A840 (2006).

13. P. P. Mukherjee and C.-Y. Wang, J. Electrochem. Soc., 154, B1121 (2007).

14. G. Wang, P. P. Mukherjee, and C.-Y. Wang, Electrochim. Acta, 52, 6367 (2007).

15. K. Kudo, T. Suzuki, and Y. Morimoto, ECS Trans., 33(1), 1495 (2010).

16. J. T. Gostick, M. W. Flower, M. D. Pritzker, M. A. Ioannidis, and L. M. Behra, J. Power Sources, 162, 228 (2006).

17. G. Inoue, T. Yoshimoto, Y. Matsukuma, and M. Minemoto, J. Power Sources, $175,145(2008)$

18. T. Springer, T. A. Zawodzinski, and S. Gottesfeld, J. Electrochem. Soc., 138, 2334 (1991).

19. A. Parthasarathy, S. Srinivasan, A. J. Appleby, and C. R. Martin, J. Electrochem. Soc., 139, 2530 (1992)

20. A. Parthasarathy, S. Srinivasan, A. J. Appleby, and C. R. Martin, J. Electrochem Soc., 139, 2856 (1992)

21. N. Nonoyama, S. Okazaki, A. Z. Weber, Y. Ikogi, and T. Yoshida, J. Electrochem. Soc., 158, B416 (2011).

22. R. Makharia, M. F. Mathias, and D. R. Baker, J. Electrochem. Soc., 152, A970 (2005) 